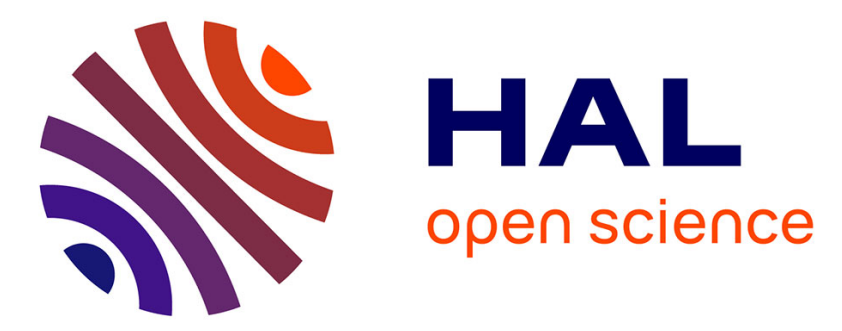

\title{
All-automatic 3D BIM modeling of existing buildings
}

Djamel-Eddine Benarab, William Derigent, David Brie, Vincent Bombardier, André Thomas

\section{To cite this version:}

Djamel-Eddine Benarab, William Derigent, David Brie, Vincent Bombardier, André Thomas. Allautomatic 3D BIM modeling of existing buildings. 15th IFIP International Conference on Product Lifecycle Management (PLM), Jul 2018, Turin, Italy. pp.56-68, 10.1007/978-3-030-01614-2_6 . hal01903840v2

\section{HAL Id: hal-01903840 \\ https://hal.science/hal-01903840v2}

Submitted on 26 Mar 2019

HAL is a multi-disciplinary open access archive for the deposit and dissemination of scientific research documents, whether they are published or not. The documents may come from teaching and research institutions in France or abroad, or from public or private research centers.
L'archive ouverte pluridisciplinaire HAL, est destinée au dépôt et à la diffusion de documents scientifiques de niveau recherche, publiés ou non, émanant des établissements d'enseignement et de recherche français ou étrangers, des laboratoires publics ou privés. 


\title{
All-automatic 3D BIM modeling of existing buildings
}

\author{
Benarab D. ${ }^{1,2,}$, Derigent W. ${ }^{1,2}$, Brie D. ${ }^{1,2}$, Bombardier V. ${ }^{1,2}$ and Thomas A. ${ }^{1,2}$ \\ ${ }^{1}$ Université de Lorraine, CRAN, UMR 7039, Campus Sciences, BP70239, 54506 Vandœuvre- \\ lès-Nancy, France \\ ${ }^{2}$ CNRS, CRAN, UMR 7039, France \\ \{djamel-eddine.benarab, william.derigent, \\ david.brie, vincent.bombardier, andre.thomas\} euniv-lorraine.fr \\ *: corresponding author
}

\begin{abstract}
In order to ensure a reliable building life cycle management, it is essential to generate an accurate and up-to-date referential mock-up that will be used for renovation, extension and maintenance. Based on this statement, we carried out, in a previous work, a research study in the sake of reconstructing a 3D CAD model from a point cloud acquired using a Lidar. This point cloud is processed automatically to detect planes and contours and to generate the 3D CAD model. However, during the life cycle of the project, different actors from different fields intervene on the building, which creates several communication conflicts, and this means a loss of time, energy and money. In order to ensure a constructive collaboration and a simplified data exchange between the different contributors in the building, we continued our work to propose, in this paper, an automatic conversion of a point cloud to a 3D BIM file. This conversion induces the passage to the standard IFC format and the integration of a valuable knowledge in it. To do so, an automatic classification of contours into architectural elements is proposed. It consists in defining a hierarchical classification rule reproducing the human reasoning for classifying the architectural elements. Then, based on the classified set of polygons, an automatic generation of 3D IFC model is proposed.
\end{abstract}

Keywords: point cloud, 3D reconstruction, mock-up, CAD, entity classification, IFC, BIM, PLM, calibration.

\section{Introduction}

Knowing that the maintenance phase represents the biggest part of the global building cost [1], it is essential to have an accurate and up-to-date referential model to approach this life cycle phase and ensure a sustainable monitoring during the whole life cycle of the building. For example, this accurate representation of the building could be used in (but not limited to) different rehabilitation phases.

Therefore, we first carried out a research study to define an automatic reconstruction process adapted to existing buildings. This one is able to generate a 3D CAD mock-up from a point cloud acquired using a Lidar 3D scanner. As will be detailed later, the 
reconstruction process automatically detects the planes of the building then the polygonal contours in each plane. The exterior polygons are considered as facades and the interior polygons are considered as architectural elements: doors, windows and others. Afterwards, these polygons constitute our CAD model that can be saved, for example, into a DXF format file.

During the life cycle of the building, different actors from different fields intervene on the building such as constructors, carpenters, plumbers, etc. In order to ensure a constructive collaboration between these actors, the BIM (Building Information Modeling) has been proposed [2,3]. This process allows an optimized collaborative work and a simplified data exchange between different actors coming from different backgrounds. This is possible thanks to the use of a common exchange format, namely, the standard IFC (Industry Foundation Classes) which is the most widely used standard currently.

For this reason, and in the sake of conceiving an all-automatic BIM modeling for the existing building, an automatic conversion of the point cloud to a standard IFC file is proposed in this paper. IFC allows to model building and construction elements (walls, windows, doors, etc.), their characteristics and relations between them. Indeed, unlike STEP and DXF formats, IFC is not limited to the sole geometry of an object but supports a richer semantic description, that we ought to use in our process by automatically generating a file composed of 3D building elements (walls, doors and windows). This requires completing our former reconstruction process with 2 additional steps:

1. The classification of the different interior polygons into building architectural elements (the exterior polygons are only known to be walls). These interior polygons can be classified into doors, windows and others;

2. The conversion of each detected building element into its corresponding IFC entity and the generation of the IFC file.

In this paper, we will start, in section 2, by presenting the research works carried out in the field of 3D reconstruction and BIM file generation. In section 3, we will illustrate a summary of our previous work of 3D reconstruction of existing buildings and introduce the first contribution, namely the polygon classification. In section 4, the automatic generation of the IFC file based on the previously classified polygons is presented. After that, several real test cases are presented in section 5, followed by a conclusion in section 6 .

\section{Related works}

Recently, several researchers have focused on developing methods for the automation of existing buildings reconstruction and BIM model generation. In terms of 3D reconstruction of building elements, the focus has been on computational geometry algorithms to extract the 3D representation of building elements through segmentation, including surface normal approaches [4], plane sweeping [5] and region growing [1, 6].

Processing a point cloud in order to reconstruct a cured 3D model is a very difficult task. The main difficulties $[2,6,7,8,9]$ are the density distribution difference of the 
point all along the point cloud, the occlusion of the building parts which are not exposed to the Lidar, the reflection of laser beams and sometimes the complexity of the building itself. In the literature, different research works sought to overcome the difficulties of as-built 3D reconstruction model. We find, for instance, several semi-automatic reconstruction methods requiring limited user intervention as in $[6,9,10,11]$. In [6] researchers proposed a method based on supervised learning in order to cope with the occlusion in interior reconstruction. In the same context of occlusion but in the exterior, researchers in [11] proposed to enhance the acquired data by a fusion of point clouds issued from laser scanning and photogrammetry. $[9,10]$ proposed to couple the point cloud with images in order to overcome reflection problems and enhance the exterior reconstruction. However, these methods remain insufficient and require a large number of parameters and their level of abstraction is often too high for the user.

In $[12,13]$ the researchers propose an automatic as-built 3D model reconstruction. These contributions have shown promising results, but they do not generate a parametric object-based model as we can find in BIM models but they generate a 3D CAD model that needs to be remodeled manually to create a BIM model.

In order to generate a BIM model, [2] proposed a semi- automatic system of the 3D reconstruction of existing buildings. Their goal is to develop a processing chain that automatically extracts the maximum amount of information from the point cloud of a building in order to integrate the result into a BIM model. To do this, they proposed to use RANSAC algorithm to segment the point cloud, the color intensity to detect the openings in each plane and the software FreeCAD to generate the IFC file.

Due to the limitations of the data collection processes as well as the complexity of as-built scenes, automated 3D modeling still presents many challenges and the majority of methods do not guarantee the accuracy of their results. Our aim, in this paper, is to propose an all-automatic and accurate 3D BIM reconstruction approach for existing buildings. For this, we start by reconstructing the 3D CAD model then we automatically generate an enriched BIM mock-up using the information parsed from the CAD model.

\section{3D CAD model reconstruction}

The 3D reconstruction process is detailed in Fig. 1. It is basically composed of 3 steps: point cloud segmentation, extraction of the different edges (or contours) of the building and ROI classification (regions of interest). Despite the classification step, the segmentation and extraction phases were already detailed in our previous works. Yet, to ensure a good understanding of the complete process, an overview of these two steps is briefly done in section 3.1, knowing that further details can be found in [7]. The new step, i.e. the classification, is described section 3.2. 


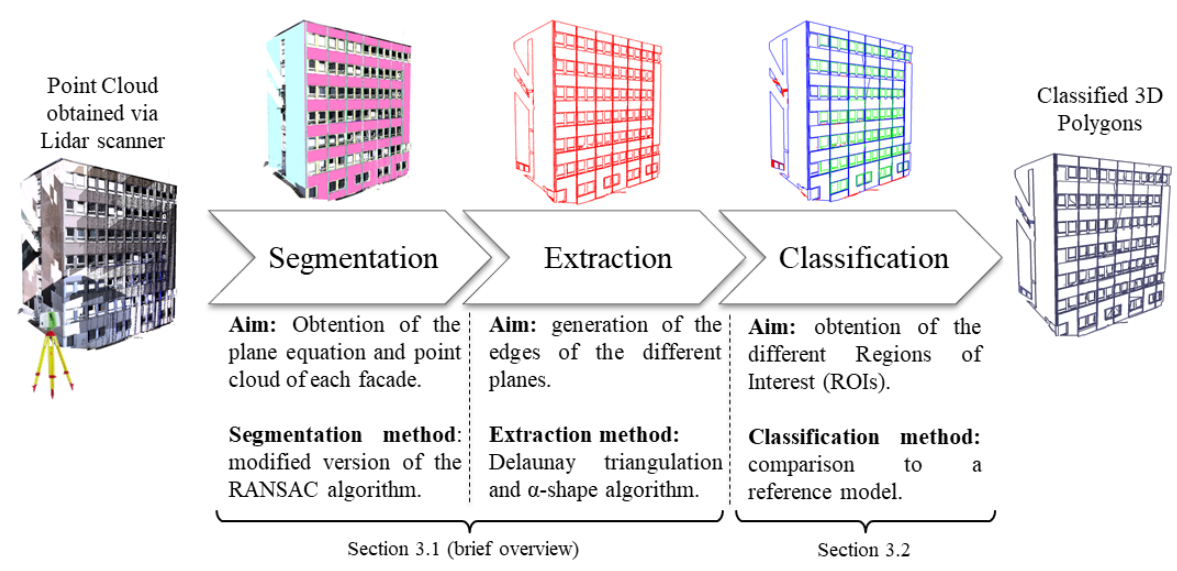

Fig. 1. The 3D reconstruction process

\subsection{Segmentation and contour extraction}

Point cloud segmentation consists in looking for the successive planes that maximize the number of consistent points (inliers). It is based on a modified version of the RANSAC algorithm, introduced by [14] in 1981. The initial algorithm is composed of three steps:

1. Proposition: In this step, the algorithm randomly chooses a sample composed of three points which implicitly define a plane equation.

2. Calculation of the cost function: The cost function consists in determining the number of inliers, which are the consistent points with the plane respecting an interval of tolerance. The other points are considered as outliers.

3. Model Ranking: Models are ranked according to the number of points that are consistent with each model. The model that maximizes the cost function is considered as the best.

These three steps can be iterated until the probability of finding a better model becomes low. A simplified (but time controlled) procedure consists of limiting the number of iterations to a fixed level. To further reduce the complexity and computation time of the algorithm, we can also consider the colorimetric data of the points cloud. Assuming the colorimetric homogeneity of the points belonging to the same geometric feature, the data point cloud is partitioned into homogeneous colorimetric clusters. Then the random sampling (step 1. of RANSAC) is selected from a single-color class. Further details can be found in [8].

The next step consists in detecting the contours of each set of co-planar consistent points. To do this, we applied the Delaunay triangulation then the $\alpha$-shape algorithm. The Delaunay triangulation is an optimal triangulation that maximizes the smallest angle of all triangles with the tendency of avoiding acute angles. The $\alpha$-shape is a generalization of the convex hull of a point set [15]. It is composed of a set of $\alpha$-exposed segments. According to [16], a segment is said to be $\alpha$-exposed if there exists an empty 
circle of radius $\alpha$ that circumcircles it. This results in a 2-D simplicial complex, which by definition is composed of vertices, edges and triangles (free and attached). A triangle edge is considered as a boundary (contour points), if it only belongs to a single triangle. The key design parameter is the radius $\alpha$. Using a constant value $\alpha$ implicitly assumes a uniform point cloud density which is not the case in real applications for which multiple stations are used. Actually, the spatial sampling step varies with the azimuth and elevation angles of the laser beam and it is minimal (i.e it has a maximal density) when the laser beam is orthogonal to the plane. The approach proposed in [7] approximates the density function of the point cloud. This allows to calculate the radius used in the $\alpha$-shape algorithm.

Once we detect the exterior and the interior contours of each facade, the Hough line detection approach is used to detect segments, which are then gathered in a closed 3D polygonal model. This is detailed in section 3.2.

\subsection{Architectural elements classification}

After the generation of the 3D geometric model of the building, a classification stage aims to provide a semantic description of the architectural elements existing inside the facade contours, in our case walls, doors and windows. For each plane, the exterior contour is considered as a wall, and interior contours are the remaining other architectural elements. For each interior polygonal contour, the identification as door or window is done by comparing the enclosing parallelepiped to the most common standard door and window geometrical models (which also are parallelepipeds).

Having defined these standard models, we measure the similarity by evaluating the quadratic distance between the enclosing parallelepiped and standard model vertices and choose the one having maximal similarity (minimal distance). The main geometrical features taken into account in order to classify entities are:

- Shape: Windows can take different shapes: square, rectangle, triangle, etc. However, doors are generally rectangular.

- Dimensions: Specific intervals are set to limit the acceptable dimensions for a window or a door. For instance, the standard model of a door is defined by a rectangle with a height between $1,7 \mathrm{~m}$ and $2,5 \mathrm{~m}$ and a length between 0,7 and $1.5 \mathrm{~m}$. More than $1,5 \mathrm{~m}$ of length can be considered as a garage. On the other hand, the standard model of windows is more general.

- Position: Position makes it possible to differentiate between a window and a door. especially the $\mathrm{Z}$ coordinate which can define whether the entity touches the floor or no.

All these criteria allow us to calculate a set of similarity measures that are compared to empirical fixed thresholds and combined in a hierarchical classification rule which reproduces thesimple human reasoning depicted in Fig.2.This process allows to classify the polygons of a plane as: wall, window, door, unknown. 


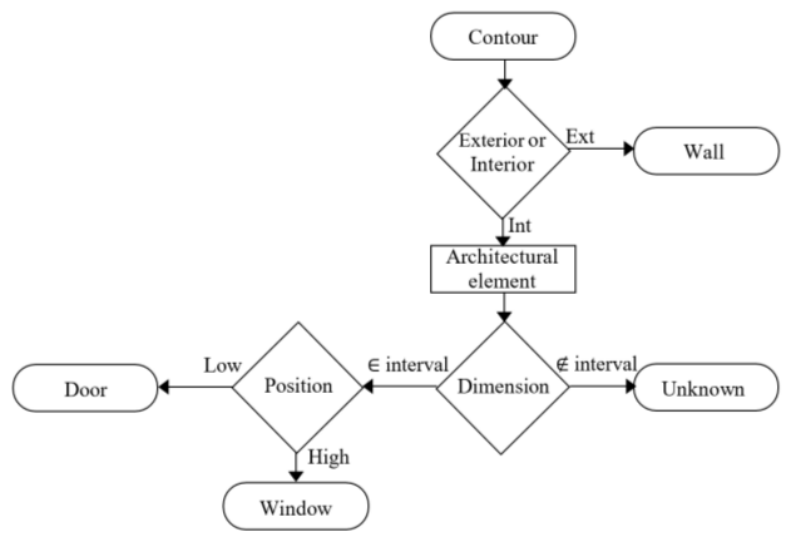

Fig. 2. A graph representation of the hierarchical classification rules

\subsection{Computation of additional features}

Once the 3D geometrical model (i.e. the set of labelled polygons) is available, several geometric, spatial and colorimetric characteristics can be computed from the point cloud and contours associated to each architecture element. From the different labelled door and window contours, we calculate the minimum surface enclosing and maximum surface enclosed parallelepipeds. For walls, the set of corresponding consistent points is used to evaluate the variance of the point clouds planarity error (granularity), and the thickness of wall in the B-rep model (see Fig. 3). In addition to this information, we export the mean plane, the minimum/maximum planes, the normal and the geometry of the plane. Moreover, some TLS generate point clouds where each point is associated with its colorimetric information. For each architectural element, the colorimetric description is description by averaging the colorimetric information over the set of consistent points.

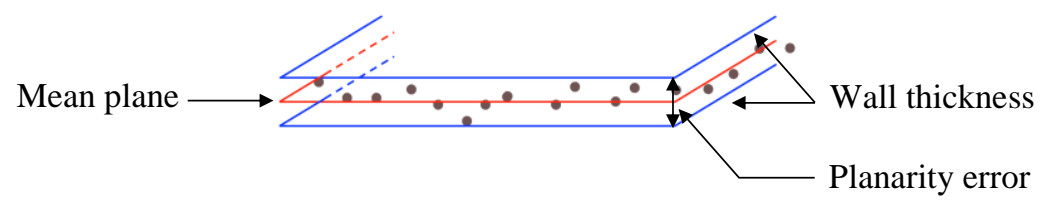

Fig. 3. Information extracted from the generated planes

\section{$4 \quad$ IFC Model generation}

The previous step generates a set of classified polygons, that remains to be converted into an IFC file. The IFC standard, currently supported and maintained by the BUILDINGSMART consortium [17], is the most popular format in the construction industry. This standard, based on STEP [18] has the needed level of expression to model 
3D shapes coupled with additional information. The IFC is a standard integrating common 3D objects with a clear semantic for the construction actors. Many building specialists import and export IFC file and many free IFC viewers are available on the market.

\subsection{Modeling types supported by the IFC BIM Standard}

Four modeling types are supported by the IFC standard: solid (B-Rep), surface, CSG, and tessellated models. To identify which modeling is the most appropriate for our purpose, several tests are realized using the files available on the Building Smart site, and different free IFC viewers. If all these different model types are authorized by the IFC standard, they are not all equally supported by BIM software tools (and especially by the free BIM viewers) available on the market. The choice of the modeling type to use has thus been guided by two constraints: 1) the chosen modeling must allow to represent surfaces issued from the contours detected by the previous steps and 2) must be compatible with a maximum number of BIM viewers, to ensure a good interoperability. As the set of classified polygons does not include the needed information to construct a building mesh, the tessellation modeling is not adapted to our use case. The remaining modeling modes has been tested on 12 different BIM viewers (11 among these are free). This list is based on our knowledge of the BIM offers and on listings available on the web ${ }^{1}$. Results presented in the table Tab. 1 show that the most interoperable modeling is the B-Rep one, compatible with all modelers and is thus the one chosen in our approach.

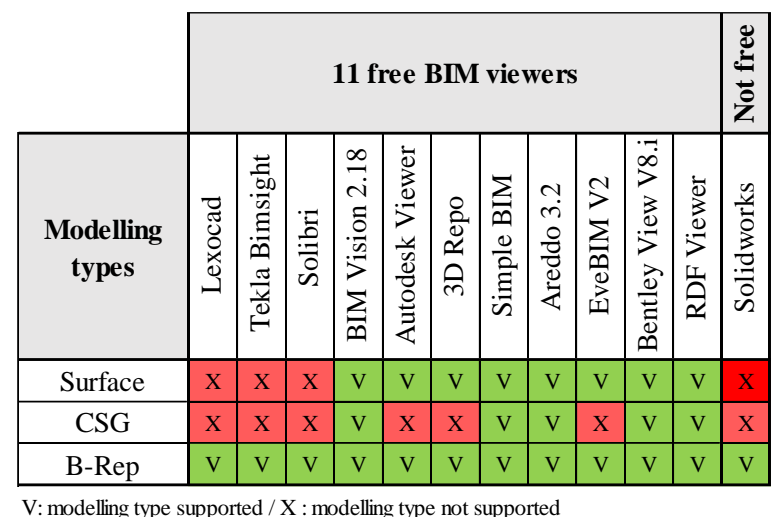

Tab. 1. Results of experimentations conducted on modeling types and BIM viewers.

The previous steps extract for each building element $E$ (facade, window or door) its contour $C$, its normal vector $N$ as well as a planarity value $P$, that corresponds to the maximum distance between the points belonging to the plane and the plane equation. As a result, the automatic IFC generation is decomposed into 2 sub-steps, 1 ) transform

\footnotetext{
${ }^{1}$ https://www.hexabim.com/blog/qui-a-teste-ces-15-viewers-ifc-gratuits
} 
the wireframe structure into a B-Rep model, and 2) create the IFC objects needed to represent facades, windows and doors, as depicted in Fig. 4.

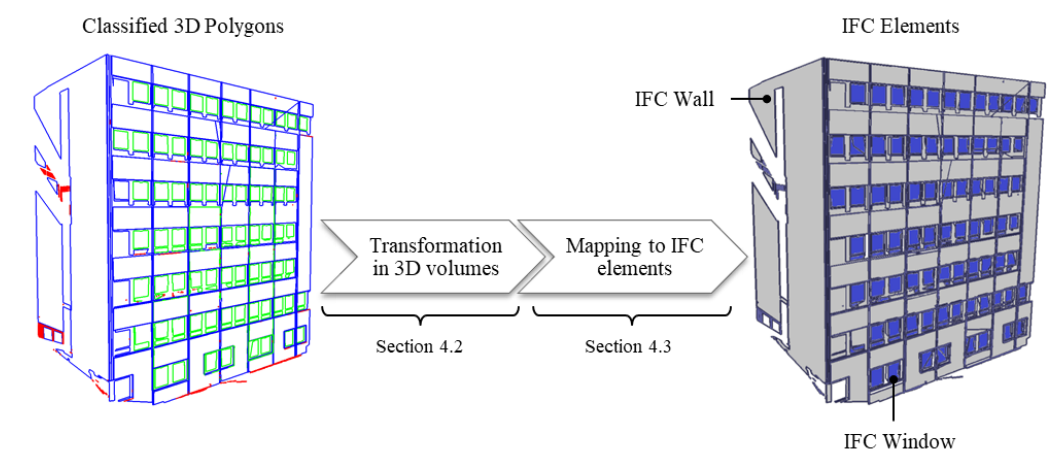

Fig. 4. IFC Model generation

\subsection{Generation of the B-Rep model}

In fact, the creation of an IFC element requires first the creation of the B-Rep elements needed to define its 3D shape. The complete process is described in Fig. 5. The final building model is not necessarily "closed", i.e. some surfaces could be missing. In fact, it is very difficult to do a complete scan of all the surfaces of a building because of diverse constraints related to accessibility and cost. As a result, building a single B-Rep for all the building is not achievable. We instead build a set of small B-Reps, one per facade, window or door, the whole set representing the final building model. This is completely in line with the IFC modeling philosophy.

Each building element $E$ (facade, door and window) first undergoes a geometric process as illustrated in Fig 5.a. This algorithm is composed of two procedures:

1. Creation of duplicated and linking lines for the considered contour: the lines of the contour $C$ are duplicated and moved along the normal vector $N$ from a distance $P$. Then linking lines, joining vertices of both contours, are constructed. They are required to form a closed wireframe shape of the building element. Using $P$ is a mean to add visual information to the final BIM model that could be used later by an engineer, in the case of a building external insulation for example. For instance, in our case, $P$ is the planarity error calculated using the associated sub-point cloud.

2. Creation of the faces $F$ and volume $V$ of the final B-Rep model.

\subsection{Mapping to IFC elements}

Once done, the second step is to associate the BRep model with an IFC element. In our case, four IFC elements are considered IFCBuilding that contains: IFCWall for facades, IFCWindow for windows and IFCDoor for doors. After the execution of the whole process, the final IFC file contains a list of all facades, doors and windows detected on the initial point cloud, and reconstructed as IFC elements as shown in 5.b. 


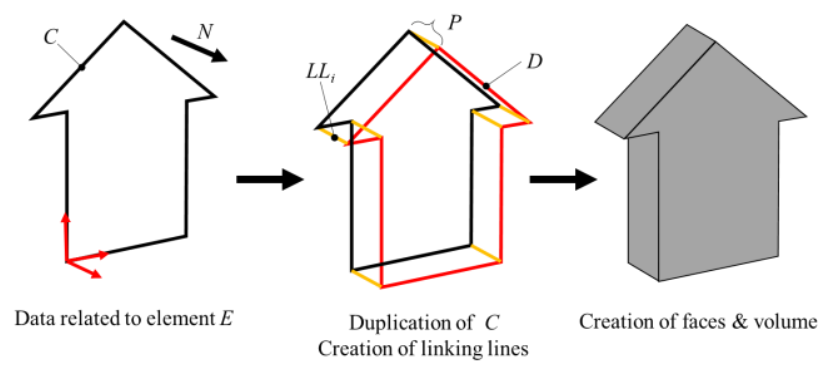

(a) Creation of the B-Rep Model of a building element $E$

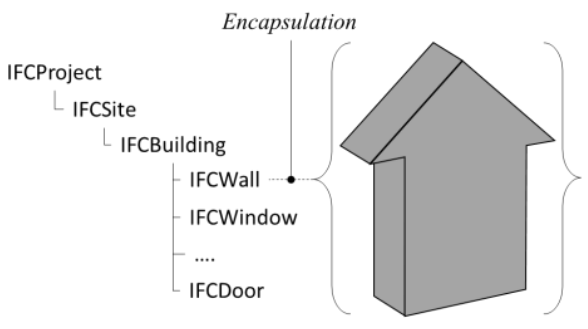

(b) Encapsulation of the B-Rep Model in an IFC structure

Fig. 5. Creation of the B-Rep Model of a building element $E$

\section{$5 \quad$ Two real-life test cases}

A set of experiment has been conducted on different point clouds coming from different sites. In the following, 2 experiments are presented. The first one is a house (calles Liezey's house) and the second one is a small co-ownership accommodation (called La Bresse's co-ownership). Our objective is to generate automatically an accurate 3D BIM mock-up for the two buildings that will be used for thermal insulation purposes.

The point cloud acquisition is carried out using the TLS Leica C10. 3D CAD reconstruction (point cloud segmentation, contour extraction) is made using ModiBuilding software (developed by the University of Lorraine). The prototype of the conversion from CAD to IFC model is implemented with Matlab software. The tests are conducted on a 6 core Apple pro equipped with an NVIDIA graphical processor unit (GPU).

Fig. 6.a illustrates the complete experiment for the Liezey's house whereas Fig. 6.b only shows the final IFC model obtained for La Bresse's co-ownership. In table 2 we present an evaluation of the architectural elements classification process carried out on the two test buildings. 

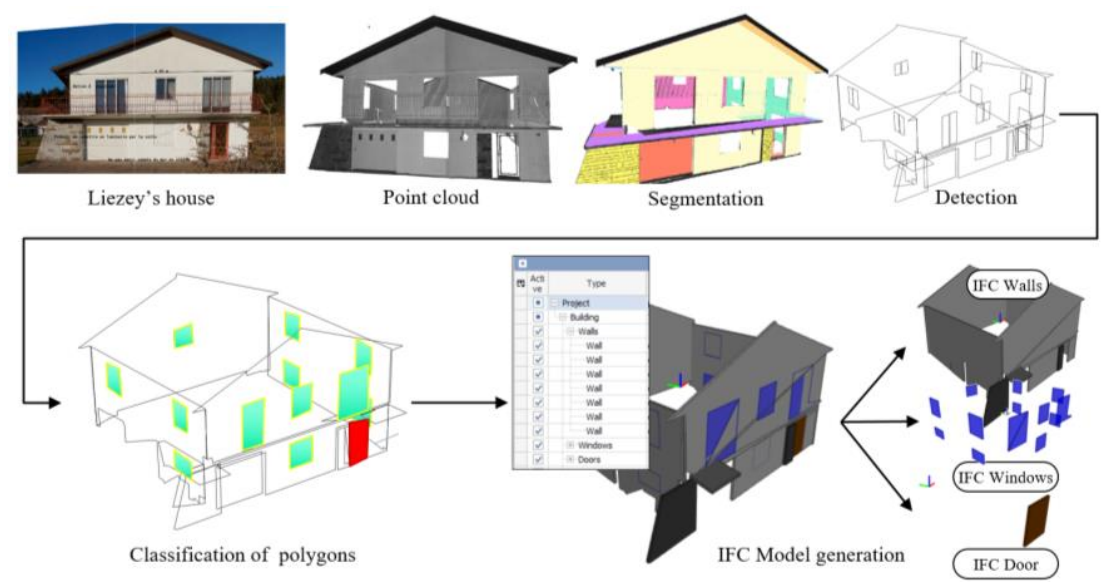

(a) Illustration of the complete process on Liezey's house

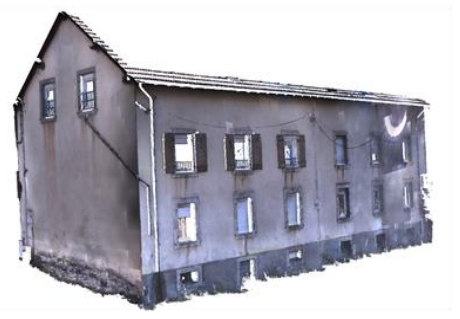

Point cloud of La Bresse's collective

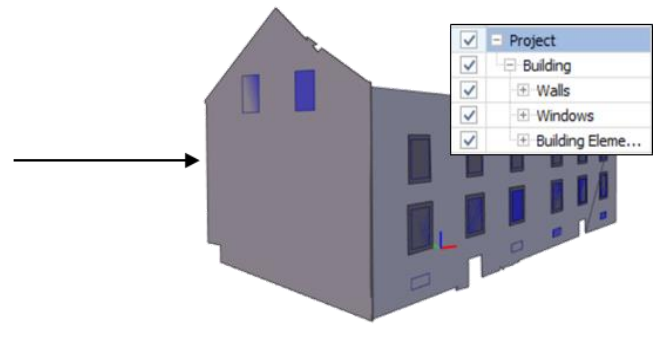

IFC Model obtained

(b) IFC Model obtained from the point cloud of La Bresse's co-ownership

Fig. 6. Experiment realized on Liezey's house and La Bresse's co-ownership

\begin{tabular}{lcc}
\hline Object & Liezey & La Bresse \\
\hline Number of windows & 13 & 18 \\
$\%$ of classified windows & $100 \%$ & $100 \%$ \\
Number of doors & 2 & 2 \\
$\%$ of classified doors & $50 \%$ & $0 \%$ \\
\hline
\end{tabular}

Tab. 2. Evaluation of the architectural elements classification process

The windows in the two buildings were well classified because they fit the standard form and dimensions of windows. However, the door of the garage in Liezey's house could not be classified because it does not have the form of a standard door. In this case, an adaptation of the classification algorithm is needed. On the other hand, the two small doors in the big facade of La Bresse's co-ownership were classified as unknown because they do not respect the lowest tolerated dimensions of a regular door. In fact, only 
half of the two doors were scanned, and another occluded half is underground and accessible using stairs. In order to detect and classify these doors, the acquisition step need to be adapted and completed with two additional stations to scan the underground part.

The table 3 presents an order of magnitude of the computation time of the reconstruction process for the two buildings. In the two cases, we didn't use the raw point cloud acquired with TLS, we applied a sampling method to lighten the point cloud and the computation time. Plane segmentation is based on the iterative algorithm RANSAC which is time consuming. Contour extraction took more time in La Bresse co-ownership because it is an old building which means a lot of planarity errors that creates false polygons to be analyzed and removed. The computation time needed for the architectural elements classification and the IFC model generation is within few seconds. Both IFC models were successfully opened using every BIM Viewers listed previously, except with SimpleBIM. The origin of this error has not been investigated yet.

\begin{tabular}{lcc}
\hline Time computation & Liezey & La Bresse \\
\hline Point cloud acquisition & $9 \mathrm{M}$ points $(1$ day $)$ & $8 \mathrm{M}$ points $(1$ day $)$ \\
Plane segmentation & $50 \mathrm{mn}$ & $45 \mathrm{mn}$ \\
Contour extraction & $55 \mathrm{mn}$ & $58 \mathrm{mn}$ \\
Classification & $<1 \mathrm{~s}$ & $<1 \mathrm{~s}$ \\
\hline
\end{tabular}

Tab. 3. Computation time of 3D reconstruction process for the two buildings

\section{Conclusion}

In this paper, a 3D BIM-oriented automatic modeling of existing buildings was presented. This process, starting from the building point cloud, is able to automatically generate 3D IFC models, fully compatible with available free BIM viewers. Our previous work has been completed by two other modules related to the classification of polygons and the IFC model generation. This improved process has been tested on numerous case studies, of which two were presented here. Experiments proved that the process is reliable but still time-consuming. However, because it is fully automatic, it may be launched in hidden time, during nights for example.

The process is for the moment limited to plane reconstruction, meaning that round shapes are discretized into numerous planes during the reconstruction process, which is not optimal. Nevertheless, this is not an important problem for applications related to building renovation of classic buildings, which is our main application target.

\section{Acknowledgments}

This research work has been carried out within the framework of the project ADEME ITE+ (External Thermal Insulation). The authors want to thank the French Environment and Energy Management Agency (ADEME) for their financial support. A special acknowledgement goes to the University of Lorraine for the use of the Modibuilding Software. 


\section{$8 \quad$ References}

1. Andrey, D., Mani, G.: Segmentation of building point cloud models including detailed architectural/structural features and MEP systems. Automation in Construction 51, 32-45 (2015). DOI: 10.1016/j.autcon.2014.12.015

2. Hélène, M.: From point cloud to building information model (BIM). 3D semi-automatic reconstruction of existing buildings. University of Strasbourg (2017).

3. Thomson, C., Boehm, J.: Automatic Geometry Generation from Point Clouds for BIM. Remote Sensing 7(9), 11753-11775 (2015). DOI: 10.3390/rs70911753

4. Barnea, S., Filin, S.: Segmentation of terrestrial laser scanning data using geometry and image information. ISPRS J. Photogramm. Remote Sens 76, $33-48$ (2013). DOI: 10.1016/j.isprsjprs.2012.05.001

5. Budroni, A., Boehm, J.: Automated 3D reconstruction of interiors from point clouds. Int. J. Archit. Comput 8, 55-73 (2010). DOI: 10.1260/1478-0771.8.1.55

6. Adan, A., Huber, D.: 3D reconstruction of interior wall surfaces under occlusion and clutter. In Proceedings of the IEEE International Conference on 3D Imaging, Modeling, Processing, Visualization and Transmission (3DIMPVT), pp. 275-281, China (2011). DOI: 10.1109/3DIMPVT.2011.42

7. Brie, D., Bombardier, V., Baeteman, G., Bennis A.: Local surface sampling step estimation for extracting boundaries of planar point clouds. ISPRS Journal of Photogrammetry and Remote Sensing (119), 309-319 (2016). DOI: 10.1016/j.isprsjprs.2016.06.006

8. Benis, A.: Contribution to the 3D reconstruction of buildings using point cloud of terrestrial laser scanning. University of Lorraine (2015).

9. Frueh, C., Jain, S. et Zakhor, A.: Data processing algorithms for generating textured 3d building facade meshes from laser scans and camera images. International Journal of Computer Vision, 61(2): 159-184, 2005. DOI: 10.1023/B:VISI.0000043756.03810.dd

10. S. Pu et G. Vosselman : Building facade reconstruction by fusing terrestrial laser points and images. ISPRS Journal of Photogrammetry and Remote sensing, 64:2525-4542, 2009. DOI: $10.3390 / \mathrm{s} 90604525$.

11. Jan Böhm, Susanne Becker et Norbert Haala : Model refinement by integrated processing of laser scanning and photogrammetry. Proceedings of the 3D Virtual Reconstruction and Visualization of Complex Architectures (3D-Arch), 2007.

12. Xiong, X., Adan, A., Akinci, B., Huber, D.: Automatic creation of semantically rich 3D building models from laser scanner data. Autom. Constr 31, 325-337 (2013). DOI: 10.1016/j.autcon.2012.10.006

13. Jung, J., Hong, S., Jeong, S., Kim, S., Cho, H., Hong, S., Heo, J.: Productive modeling for development of as-built BIM of existing indoor structures. Autom. Constr 42, 68-77 (2014). DOI: 10.1016/j.autcon.2014.02.021

14. Fischler, M.A., Bolles, R.C.: Random sample consensus: A paradigm for model fitting with applications to image analysis and automated cartography. Communications of the ACM 24, 381-395 (1981). DOI: 10.1145/358669.358692

15. Edelsbrunner, H., Kirkpatrick, D.G., Seidel, R.: On the shape of a set of points in the plane. IEEE Trans. Inf. Theory 29, 551-559 (1983). DOI: 10.1109/TIT.1983.1056714

16. Edelsbrunner, H., Mucke, E.P.: Three-dimensional Alpha Shapes. ACM Trans. Graph 13(1), 43-72 (1994). DOI: 10.1145/174462.156635

17. ISO.: Industry Foundation Classes (IFC) for data sharing in the construction and facility management industries. (2013).

18. Pratt, M.J.: ISO 10303, the STEP standard for product data exchange, and its PLM capabilities. Int. J. Prod. Lifecycle Manag 1(1), 86 (2005). DOI: 10.1504/IJPLM.2005.007347 